\title{
Mechanism of gas molecule transport through erythrocytes' membranes by kinks-solitons
}

\author{
P. V. Mokrushnikov ${ }^{1}$, V. Ya. Rudyak ${ }^{1,2}$, E. V. Lezhnev ${ }^{1}$ \\ ${ }^{1}$ Novosibirsk State University of Architecture and Civil Engineering, \\ Leningradskaya, 113, Novosibirsk, 630008, Russia \\ ${ }^{2}$ Novosibirsk State University, Pirogova, 1, Novosibirsk, 630090, Russia \\ pavel.mokrushnikov@bk.ru, valery.rudyak@mail.ru, lionlev@yandex.ru
}

PACS 87.16.Dg, 87.16.Uv, 87.15.He

DOI 10.17586/2220-8054-2021-12-1-22-31

\begin{abstract}
A model of kinks appearance in the lipid bilayer membrane of erythrocytes, which are responsible for gas molecule transport, in particular, oxygen, is proposed. It was shown that the kinks arise due to the simultaneous action of transverse and tensile longitudinal mechanical stresses compressing the membrane. This model explains the membrane's permeability sharp increase for gases during an erythrocyte passage through the microcapillary network with the compressive transverse mechanical stresses sharply increasing in its membrane. It was found that the equation of kinks motion has a soliton solution, so that a kink-soliton is formed in the bilayer of the erythrocyte membrane. The developed model is consistent with the previously experimentally established fact that the native erythrocyte membranes in the bloodstream undergo a structural transition, when small changes in blood $\mathrm{pH}$, hormone concentration, and temperature dramatically change the conformation of the biomembranes and its functions by changing the mechanical stress field in the biomembrane.
\end{abstract}

Keywords: biomembrane, diffusion, kinks, mechanical stress in biomembranes, microcapillary, nanosystem, erythrocyte.

Received: 15 November 2020

Revised: 20 December 2020

\section{Introduction}

Gas molecule transfer $\left(\mathrm{O}_{2}, \mathrm{CO}, \mathrm{CO}_{2}, \mathrm{NH}_{4}\right)$ through an erythrocyte's membranes is an important physiological process. Biomembranes usually consist of two lipids layers, where proteins are embedded. This is a typical nanosystem, since the thickness of lipid layers does not usually exceed $10 \mathrm{~nm}$. The structural state of the biomembrane is very complex. In the normal state, the membrane is in a liquid crystalline state, but a phase transition to the gel phase can occur in it, depending on the external conditions. In general, lipids perform rather complex movements, including lateral diffusion, rotational movement around the axis, and some others. The lateral diffusion coefficient of spin-labeled or fluorescent phospholipid probes can be analyzed by several methods: electronic paramagnetic resonance, nuclear magnetic resonance and fluorescence. The root-mean-square displacement of such a probe is determined by the Einstein relation: $\vec{l}^{2}=4 D t$ (two-dimensional system). In this case, the diffusion coefficient values were found to be quite high: $D=10^{-11}-10^{-12} \mathrm{~m}^{2} / \mathrm{s}[1]$.

Gas molecule transport through the biomembrane should be determined by the same mechanisms as the diffusion probe. It was experimentally established that the erythrocyte's membrane permeability for gas molecules is not lower than the water layer permeability of the same thickness [2]. This is rather strange, since the dynamic viscosity of the lipid bilayer, which forms most of the erythrocyte membrane surface, is from 30 to $100 \mathrm{mPa} \cdot \mathrm{s}$, and water is about $1 \mathrm{mPa} \cdot \mathrm{s}$. Indeed, the diffusion coefficient $D$ of the spherical particle with radius $r$ is determined by the EinsteinStokes formula: $D=k T / 6 \pi \mu r$, where $k$ is the Boltzmann constant, $T$ is the temperature, and $\mu$ is the medium viscosity coefficient. This leads to the fact, that diffusion resistance of the lipid bilayer should also be greater than the corresponding value for the same water layer. The data of measurements of the oxygen molecules permeability through monolayer artificial lipid membranes are in agreement with this conclusion. Their permeability is shown to be significantly lower than that of a water layer of similar thickness $[3,4]$.

The observed facilitated diffusion of oxygen molecules are attempted to explain by the fact that the main stream of oxygen molecules passes through the proteins' membrane of aquaporin AQP1 in a number of papers [5-8]. Currently, aquaporins have also been associated with the transport of $\mathrm{CO}, \mathrm{CO}_{2}, \mathrm{O}_{2}, \mathrm{NH}_{4}$ [9-11]. Such a mechanism actually takes place, but it does not explain the sharp increase in the gas molecule's transport through the membrane during the erythrocyte's passage through the microcapillary chain and into lungs alveoli. There must also be a mechanism (or mechanisms) regulating this flux.

Facilitated gaseous diffusion is necessary through the erythrocyte's membranes when the hemoglobin of the erythrocyte is saturated with oxygen in the lungs capillaries. It is known that in this case, about a third of the oxygen, carried by it, penetrates into the erythrocyte cytoplasm in a quarter of a second [12]. There is no known mechanism that 
allows such a large amount of oxygen to be transferred through the membrane in such a short time. There is another problem with gas exchange in the cardiovascular system. Gas exchange is not desirable in a case, when erythrocytes pass through the arteries and veins. It should occur during the passage of erythrocytes through microcapillaries, penetrating tissues and through the lungs alveoli. Otherwise, oxygen entered the bloodstream, increases the concentration of reactive oxygen species in the blood, which leads to an increase in lipid peroxidation of the cell membranes in blood vessel walls.

It was previously suggested that the gaseous diffusion in the lipid bilayer can occur with the help of kinks running along the hydrocarbon chains of phospholipids, that is, bends arising in the hydrocarbon chains [13, 14]. It was believed that kinks arise due to the energy of thermal vibrations of phospholipids. While paying tribute to such a mechanism, one should note its obvious shortcomings. First of all, the indicated fluctuating nature of kink formation determines their obvious instability. It is unclear how the kinks appearance synchronization occurs in the hydrocarbon chain, which should determine the directional transport of gas molecules by kinks along the hydrocarbon chain.

The aim of this paper is to study the mechanism of the kinks' appearance in the hydrocarbon chain of phospholipids. It is shown that they arise as a result of mechanical transverse compression of the erythrocyte membrane during its passage through the microcapillary layer and through the lungs alveoli. The mathematical model describing the kinks' evolution is formulated and it is established that not simple kinks, but the kinks-solitons are formed. The models of kink-solitons, running along the infinite hydrocarbon chain of the erythrocyte membrane lipids and along a real chain of finite length, are analyzed.

\section{Equation of a hydrocarbon chain motion}

In order to study the transport of gas molecules by erythrocytes, it is first necessary, to analyze their passage through the corresponding membrane, through the corresponding lipid bilayer. So, we should consider a hydrocarbon chain section of a phospholipid molecule with saturated bonds. It consists of $\mathrm{n}$ carbon groups and the distance between them is determined by the valence bonds and the corresponding angles. A section of such a hydrocarbon chain of three $\mathrm{CH}_{2}$-groups (groups $i, i+1, i-1$ ) is shown in Fig. 1, where $\theta_{i}$ is the bond angle, and $\varphi_{i}$ is the angle of internal rotaton. The bond lengths and bond angles $\theta_{i}$ remain constant (Fig. 1). The polymer molecule conformation is determined by the angles of rotation around $\mathrm{C}-\mathrm{C}$ bonds, or angles of internal rotation [15]. These are the angles between two planes formed by bond pairs $(i-1, i)$ and $(i, i+1)$.

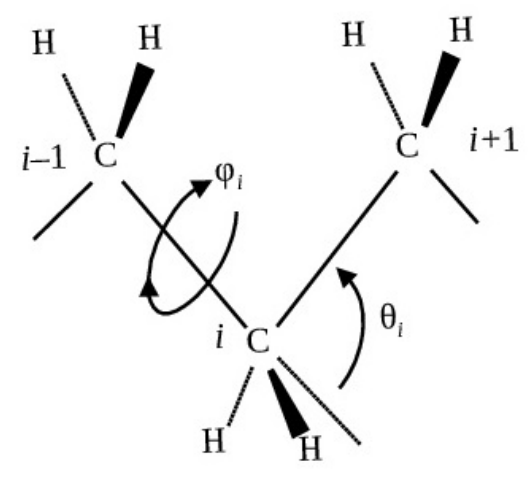

FIG. 1. A hydrocarbon chain section with saturated bonds: $i$ is a number of $\mathrm{CH}_{2}$-group of atoms, $\theta i$ is the bond angle, $\varphi_{i}$ is the angle of internal rotation

The erythrocytes roll up into cylinders, passing through microcapillaries [16]. If there are no stretching regions in the lipid bilayer, then the double hydrocarbon tails of phospholipid molecules in the biomembrane are in a completely trans-configuration. All angles are zero, and the hydrocarbon chain is a linear structure in the trans-conformation. This is illustrated in Fig. 2a, which is a side view of the double hydrocarbon tails of phospholipid molecules in the trans-configuration.

On the contrary, in the general case, when erythrocytes pass through the microcapillary, longitudinal mechanical tensile stresses $G$ and mechanical compressive stresses $\mathbf{P}$ increase in the direction transverse to the membrane plane in their plasma membranes (see Fig. 2b). The hydrocarbon tail loses its stability, its links begin to rotate under the action of the transverse mechanical compression stress and the longitudinal mechanical tensile stress. This leads to the bending of the phospholipid chain and a kink formation. As a result of membrane compression, its thickness decreases by $h=0.127 \mathrm{~nm}$ (see Fig. 2b). 

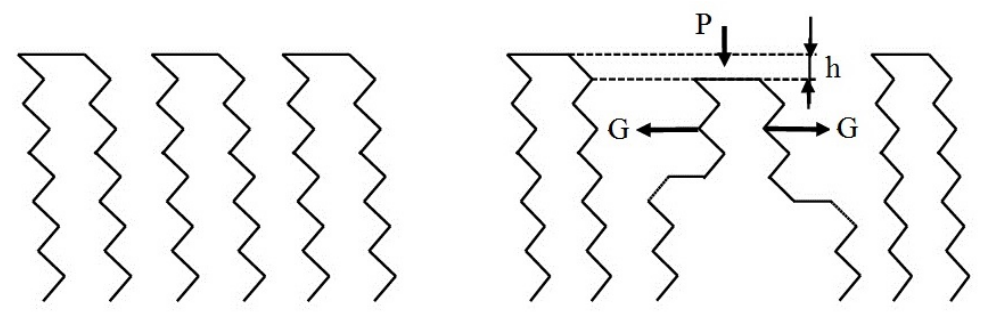

FIG. 2. Side view of double hydrocarbon tails of phospholipid molecules in a biomembrane, which are in trans-configuration (a) and in the presence of transverse and longitudinal mechanical stresses (b)

The appearance of a kink is due to the rotation of $\mathrm{CH}_{2}$ groups around the OX axis by an angle $\Psi_{i}$ (see Fig. 3), which arises under the action of transverse and longitudinal mechanical stresses. The rotation of each $\mathrm{CH}_{2}$-group is determined by the forces moment $\mathbf{N}_{i 1}$, created by the interaction between this hydrocarbon chains group of neighboring phospholipid molecules. In addition, it is necessary to take into account the forces moment $\mathbf{N}_{i 2}$, created during steric interaction of hydrogen atoms during rotation of $\mathrm{CH}_{2}$-groups $i$ and $(i+1)$ and groups $i$ and $(i-1)$ around $\mathrm{C}-\mathrm{C}$ bonds. Finally, one should take into account the forces moment $\mathbf{N}_{i 3}$, created by the interaction of the membrane electric field and the induced dipole moment of the $\mathrm{C}-\mathrm{C}$ bond. Thus, the equation of the $\mathrm{i}$-th group motion has the following form:

$$
J \frac{\partial^{2} \Psi_{i}}{\partial t^{2}}=\mathbf{N}_{i 1}+\mathbf{N}_{i 2}+\mathbf{N}_{i 3}
$$

where $J$ - an inertia moment $i$-th $\mathrm{CH}_{2}$-groups around the axis $\mathrm{OX}$ (Fig. 1).

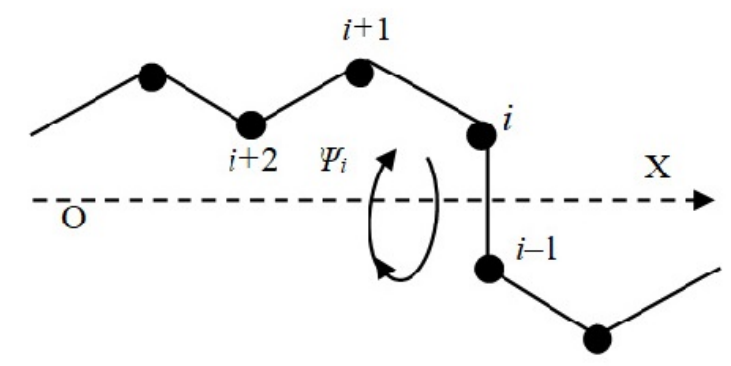

FIG. 3. The kink is moved by turning the $\mathrm{CH}_{2}$-group number $i$ around the axis rotation $\mathrm{OX}$ by the angle $\Psi_{i}$. The OX axis is directed along the hydrocarbon chain

Let us consistently consider the moments $\mathbf{N}_{i 1}, \mathbf{N}_{i 2}, \mathbf{N}_{i 3}$ in eq. (1). In the trans-configuration of the hydrocarbon chain $\Psi_{i}=0$. On the other hand, in gauche(+)-trans-gauche(-)-configurations $\Psi_{i}=\pi$. The forces moment, created by the interaction of neighboring hydrocarbon chains $\mathbf{N}_{i 1}$ is maximum at $\Psi_{i}=\pi / 4,3 \pi / 4,5 \pi / 4$ and $7 \pi / 4$ and is equal to zero at $\Psi_{i}=0, \Psi_{i}=\pi / 2, \pi$ and $3 \pi / 4$. Indeed, at $\Psi_{i}=0$ or $\Psi_{i}=\pi$, the arm of force $\mathbf{F}$ acting between the $i$-th $\mathrm{CH}_{2}$-group and the hydrocarbon chains of neighboring phospholipid molecules in the process of rotating around OX axis rotation is equal to zero. When $\Psi_{i}=\pi / 2$ or $3 \pi / 2$, the force $\mathbf{F}$ is equal to zero. Thus, this forces moment is equal to: $\mathbf{N}_{i 1}=K_{F} \sin \left(2 \Psi_{i}\right)$, where $K_{F}=F l$, here $l$ is the length of the $\mathrm{C}-\mathrm{C}$ bond, and $\mathbf{F}$ is the force, tending to rotate the $\mathrm{CH}_{2}$-group $i$ around the $\mathrm{C}-\mathrm{C}$ bond by $\Psi_{i}$ angle (see Fig. 3). This force can be estimated using Young's modulus of longitudinal tension $\mathbf{E}_{\|}$of a biomembrane [17]. Further, it will be assumed that $K_{F}=$ const.

There is an electric field with strength $E$, which causes the polarization of the $\mathrm{C}-\mathrm{C}$ bonds of phospholipid molecules in the native membrane of the erythrocyte. Each $\mathrm{C}-\mathrm{C}$ bond acquires an electric dipole moment. Let us denote the electric dipole moment between carbon atoms $(i-1)$ and $i$ as $\mathbf{p}_{i-1}$, between $i,(i+1)$ as $\mathbf{p}_{i}$, and between $(i+1),(i+2)$ as $\mathbf{p}_{i+1}$. Two positions of these three dipoles are shown in Fig. 4 with arrows, when a kink passes through them at $\Psi_{i}=0$ and $\Psi_{i}=\pi$. The dashed lines indicate the force lines of the electric field strength $\mathbf{E}$ in the membrane. The interaction energy of the electric dipole $\mathbf{p}$ with the electric field is equal to $W_{0}=-\mathbf{p} \cdot \mathbf{E}=-p E \cos (\alpha)$, where $\alpha$ is the angle between $\mathbf{E}$ and $\mathbf{p}$ vectors. The dipole modulus equals to $p=\varepsilon_{0} \beta \cos \alpha$, where $\beta$ is the polarizability coefficient of the $\mathrm{C}-\mathrm{C}$ bond and $\varepsilon_{0}$ is the electrical constant. The energy $W_{0}$ is determined by the angle $\alpha$. When the three dipoles rotate around the OX axis from $\Psi_{i}=0$ to $\Psi_{i}=\pi$ for $\mathbf{p}_{i-1}$ dipole, the angle $\alpha$ changes from $\pi / 3$ 
to $\pi / 2$, for $\mathbf{p}_{i-1}$ dipole, the angle $\alpha$ does not change and is equal to $\pi / 3$, for $\mathbf{p}_{i+1}$ the angle $\alpha$ varies from $\pi / 2$ to $\pi / 3$. When $\Psi_{i}=\pi / 2$ for $\mathbf{p}_{i-1}$ and $\mathbf{p}_{i+1}$, the angle $\alpha=75^{\circ}$. Between the states of three dipoles $\mathbf{p}_{i-1}, \mathbf{p}_{i}, \mathbf{p}_{i+1}$ at $\Psi_{i}=\pi$ and at $\Psi_{i}=\pi$ there is an energy barrier with a height $\Delta W_{E}=\varepsilon_{0} \beta E^{2}$ created by the interaction of these three dipoles with the electric field of the membrane. Thus, it is easy to establish that the forces moment $\mathbf{N}_{i 2}$ is equal to $\mathbf{N}_{i 2}=-K E \sin \left(2 \Psi_{i}\right)$.

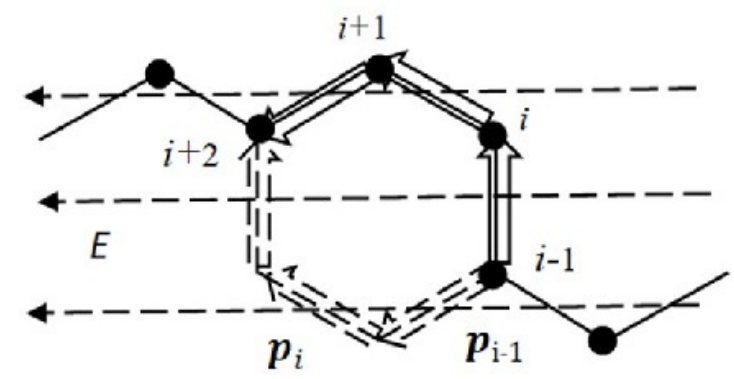

FIG. 4. Two positions of three dipoles $\mathbf{p}_{i-1}, \mathbf{p}_{i}, \mathbf{p}_{i+1}$ at $\Psi_{i}=0$ (flat dashed arrows) and $\Psi_{i}=\pi$ (flat solid arrows) in the membrane electric field $\mathbf{E}$

The force moment $\mathbf{N}_{i 3}$ is created by the $i$-th atomic group interaction with the neighboring groups $(i-1)$ and $(i+1)$ (see Fig. 3). It appears because of the barrier $\Delta E$ in the interaction energy of neighboring atomic $\mathrm{CH}_{2}$-groups, which appears during these groups rotation around $\mathrm{C}-\mathrm{C}$ bonds. The barrier arises as a result of steric repulsion of closely spaced valence unbonded hydrogen atoms of neighboring $\mathrm{CH}_{2}$-groups. The energy minimum occurs if two pairs of hydrogen atoms of neighboring $\mathrm{CH}_{2}$-groups are in a crossed state $\left(\left(\Psi_{i-1}-\Psi_{i}\right)=0\right.$ or $\left.\pi\right)$, and the maximum corresponds to shaded conformations $\left.\left(\left(\Psi_{i-1}-\Psi_{i}\right)=\pi\right)\right)$. For a saturated hydrocarbon chain, $\Delta E$ barrier changes from 12 to $17 \mathrm{~kJ} / \mathrm{mol}$, depending on the link and the length position of the hydrocarbon chain [17]. If the difference in the angles of neighboring groups rotation is small or at least less $\left.\pi / 2\left(\Psi_{i-1}-\Psi_{i}\right) \leq \pi\right)$, then we can approximately assume that the forces moments, created by the interaction of atomic group $i$ with atomic groups $(i-1)$ and $(i+1)$ are respectively equal to $\chi\left(\Psi_{i-1}-\Psi_{i}\right)$ and $\chi\left(\Psi_{i+1}-\Psi_{i}\right)$, where $\chi$ is some constant determined by the following relation: $\chi=\Delta E /(\pi / 2)^{2}$. Here $\Delta E$ is the barrier value in the energy of neighboring atomic $\mathrm{CH}_{2}$-groups interaction, which appears during these groups rotation around $\mathrm{C}-\mathrm{C}$ bonds. Therefore, the total moment turns out to be equal to $N_{i 2}=\chi\left(\Psi_{i+1}-2 \Psi_{i}+\Psi_{i-1}\right)$.

Substituting the obtained expressions for the moments into eq. (1), we obtain:

$$
J \frac{\partial^{2} \Psi_{i}}{\partial t^{2}}=\chi\left(\Psi_{i+1}-2 \Psi_{i}+\Psi_{i-1}\right)+\left(K_{F}-K_{E}\right) \sin 2 \Psi_{i}, \quad i=1,2, \ldots N
$$

where $N$ is the number of atomic groups in the simulated hydrocarbon chain.

\section{Soliton solution for the equation of a kink motion}

Let us first consider the evolution of a sufficiently long hydrocarbon chain. The number of $\mathrm{CH}_{2}$-groups is large and the chain length is much greater than the distance between them in such a chain. In order to qualitatively describe its evolution, one needs to go to the limit, directing the distance between groups to zero. In fact, this is equivalent to considering an infinite chain. So, if we denote the distance between neighboring atomic groups by 1 and put $\chi=K l^{-2}$, we will assume that $K$ remains a finite constant as $l \rightarrow 0$ (this is a kind of the thermodynamic limit transition analogue [18], if we remind that the number of groups tends to infinity). Directing the OX axis along the hydrocarbon tails, it is easy to see that eq. (2) is reduced to the sine-Gordon differential equation [19]:

$$
J \frac{\partial^{2} \Psi}{\partial t^{2}}-K \frac{\partial^{2} \Psi}{\partial x^{2}}-\left(K_{F}-K_{E}\right) \sin 2 \Psi=0 .
$$

We will find a solution to this equation in the form of $f(\xi)$ function, where $\xi=x \pm V t, x$ is the coordinate along $\mathrm{OX}$ and $V$ is the kink velocity. Substituting this solution into (3), after simple transformations we find

$$
\frac{1}{2}\left(\frac{\partial f}{\partial \xi}\right)^{2}+\frac{\left(K_{F}-K_{E}\right)}{2\left(J V^{2}-K\right)} \cos (2 f)=\gamma
$$

where $\gamma=$ const. 
Equation (4) is the equation of an oscillator moving in a potential well $U(f)=U_{0} \cos (2 f)$, where $U(f)=$ $U_{0} \cos (2 f)$, and $U_{0}=\left(K_{F}-K_{E}\right) /\left[2\left(J V^{2}-K\right)\right]$. At $\gamma<-U_{0}$ the equation has no solution. On the other hand, if $-U_{0}<\gamma<U_{0}$, then the oscillator performs periodic oscillations inside one of the potential well. Finally, for $\gamma=-U_{0}$, the solution describes a soliton, i.e. isolated perturbation tending to constant values at infinity: $\lim _{\xi \rightarrow \pm \infty} f(\xi)=$ const. Thus the soliton solution of the eq. (4) has the following form:

$$
\tan (f / 2)=\exp \left\{ \pm 2 \sqrt{\gamma}\left(\xi+\xi_{0}\right)\right\}
$$

where $\xi_{0}=$ const. The dependence of the function $f$ from $(x-V t)$ is presented in Fig. 5, here $h$ is the width of the kink-soliton.

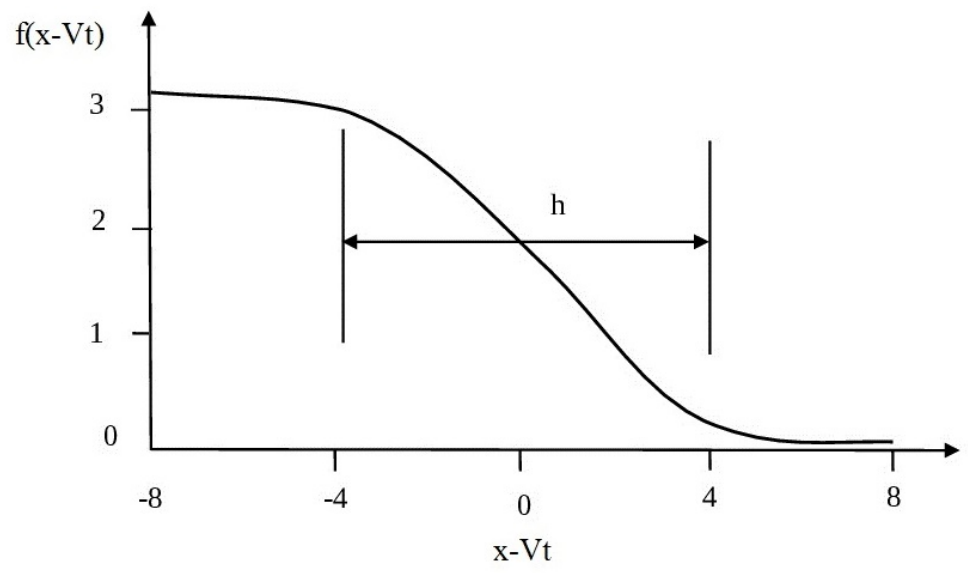

FIG. 5. The form of solution (5) for $2 \sqrt{\gamma}=V=1$

For the kinks-solitons appearance in the hydrocarbon chain, such a chain must be subjected to mechanical compression with a strictly defined value of transverse pressure $\mathbf{P}$. In this case, the phospholipid is compressed by $\Delta$, and then straightens and the kink-soliton runs along it. So, in order to do it, the work $A$ of the mechanical pressure $\mathbf{P}$, compressing the membrane in the transverse direction must be equal to the energy of the kink-soliton $W$ and the kinetic energy, transferred by the kink-soliton at the velocity $V$ of a gas molecule with mass $m$. We estimate the work $A$ using Young's modulus of transverse compression $\mathbf{E}_{\perp}$ of the biomembrane. The energy of the kink-soliton is calculated the same way, as it is done in [19]. In order to do it, we find the density of the Hamiltonian from eq. (3), and then calculate the energy of the kink-soliton in an infinite hydrocarbon chain. The width of the kink is equal to $h$ (Fig. 5). At this distance, the angle of $f$ rotation varies from 0 to $\pi$. This is done under the following conditions:

$$
\gamma=\frac{\left(K_{F}-K_{E}\right)}{2\left(J V^{2}-K\right)}, \quad \frac{E_{\perp} S \Delta^{2}}{2 L}=W \frac{m V^{2}}{2}, \quad \sqrt{\gamma}=\ln 2
$$

where $S$ is lipid cross-sectional area, and $L$ is its length.

From conditions (6), it is possible to estimate velocity of the kink-soliton, its energy $W$, and the value of critical compression $\Delta$. In order to do this, it is necessary to evaluate the constants $K, K_{F}, K_{E}$. The force $\mathbf{F}$, tending to rotate the $\mathrm{CH}_{2}$-group $i$ around the $\mathrm{C}-\mathrm{C}$ bond, can be estimated using the Young's modulus of longitudinal tension $E_{\|}$ of the biomembrane $F=\varepsilon E_{\|} S_{1}$, where $S_{1}$ is the area, occupied by the $\mathrm{CH}_{2}$-group, and $\varepsilon$ is the relative membrane elongation. Setting $\varepsilon=0.01, E_{\|}=5 \cdot 10^{8} \mathrm{~N} / \mathrm{m}^{2}, S_{1}=0.084 \mathrm{~N} \cdot \mathrm{m}^{2}, l=0.15 \mathrm{~nm}$ [17], we get $K_{F}=0.628$. $10^{-22} \mathrm{~N} \cdot \mathrm{m}$.

The polarizability is $\beta=1.25 \cdot 10^{-30} \mathrm{~m}^{3}$, and the electric field strength in the erythrocyte membrane is $E \approx$ $5 \cdot 10^{7} \mathrm{~V} / \mathrm{m}$ for C-C bonds, [17]. Substituting these values into the expression for $K_{E}=\left(2 \Delta W_{E} / \pi\right)$, we get $K_{E}=2.044 \cdot 10^{-27} \mathrm{~N} \cdot \mathrm{m}$. We estimate the constant $\chi$ by the formula $\chi=\Delta E /(\pi / 2)^{2}$, where $\Delta E$ is the value of the barrier in the interaction energy of neighboring atomic $\mathrm{CH}_{2}$-groups, which appears when these groups rotate around $\mathrm{C}-\mathrm{C}$ bonds. Taking $\Delta E=12.5 \mathrm{~kJ} / \mathrm{mol}$, we find that $\chi=0.843 \cdot 10^{-20} \mathrm{~N} \cdot \mathrm{m} / \mathrm{rad}$. Therefore the constant $K=\chi l^{2}$ is equal to the following value $K=1.897 \cdot 10^{-40} \mathrm{~N} \cdot \mathrm{m}^{3} / \mathrm{rad}$. The inertia moment of the $\mathrm{CH}_{2}$-group around the OX axis rotation (Fig. 3) $J=M l^{2}$, where $M$ is the mass of the $\mathrm{CH}_{2}$-group. Substituting the corresponding value values, we find $J=0.544 \cdot 10^{-45} \mathrm{~kg} \cdot \mathrm{m}^{2}$. The velocity of the kink-soliton is $V=591 \mathrm{~m} / \mathrm{s}$. 
The energy of the kink-soliton W is calculated from the density of the Hamiltonian $\mathrm{H}$ corresponding to the eq. (3):

$$
W=\int_{-\infty}^{+\infty} H d x, \quad H=\frac{1}{2}\left[j\left(\frac{\partial f}{\partial t}\right)^{2}+\frac{K}{l}\left(\frac{\partial f}{\partial x}\right)^{2}-\frac{\left(K_{F}-K_{E}\right)}{l} 2 \sin ^{2} f\right],
$$

where $j=\left(M l^{2}\right) / 2$ is the linear density of the inertia moment of the hydrocarbon chain. Substituting solution (5) and relations (6) into (7), we find:

$$
W=2 j V^{2} \sqrt{\gamma}+\frac{2 K}{l} \sqrt{\gamma}-\frac{\left(K_{F}-K_{E}\right)}{l \sqrt{\gamma}}=\frac{2.772 K}{l h} .
$$

Thus, the energy of the kink-soliton depends only on the barrier value $\Delta E$ of the interaction energy of neighboring atomic $\mathrm{CH}_{2}$-groups, which appears during these groups rotation around $\mathrm{C}-\mathrm{C}$ bonds. Substituting the corresponding values, we obtain $W=2.703 \cdot 10^{-20} \mathrm{~J}$, and the critical compression of the phospholipid is equal to $\Delta=1.28 \mathrm{~nm}$.

\section{Modeling the kinks-solitons in silico motion}

In the previous section, a kink was shown to arise in a hydrocarbon chain as a result of its mechanical deformation. Its dynamics are described by a soliton (5) in the approximation of an infinite chain: the kink-soliton moves along the hydrocarbon chain. Such kinks-solitons, which appear in the lipid bilayer as a result of structural changes in biomembranes, can indeed determine the real mechanism of gas molecules transporting. At the same time, it is obvious that in a real situation the hydrocarbon chain has a finite size. Do the kinks-solitons form in such a chain, and if so, how do their characteristics depend on the parameters of the system modeled? This section is devoted to answering these questions. In silico simulation of the dynamics of a finite-length acyl chain is performed here. Situations are simulated when the number of links in the hydrocarbon chain is limited and when three of the bonds are unsaturated $(\mathrm{C}=\mathrm{C})$.

Equation (2) of motion about the axis of the $\mathrm{OX}$ ith $\mathrm{CH}_{2}$-group has the form:

$$
J \frac{\partial^{2} \Psi_{i}}{\partial \tau^{2}}-A\left(\Psi_{i+1}-2 \Psi_{i}+\Psi_{i-1}\right)-B \sin 2 \Psi_{i}=0, \quad i=1,2, \ldots, N .
$$

Here, the time is normalized to the time $\tau_{0}=10^{-12} \mathrm{~s}$, it is determined by the interaction time of the neighboring $\mathrm{CH}_{2}$-groups of the hydrocarbon chain, which is of the order of $l / V$. It is easy to establish that this time in this case is $0.26 \cdot 10^{-12} \mathrm{~s}$. The coefficient $A=\left(\chi \tau_{0}^{2} / J\right)$ included in (9) is determined by the forces moment acting between neighboring $\mathrm{CH}_{2}$-groups as a result of steric interaction of hydrogen atoms as they rotate around $\mathrm{C}-\mathrm{C}$ bonds. For the erythrocyte membrane, $A=15.5$ for a single $\mathrm{C}-\mathrm{C}$ bond. For a link with a double $\mathrm{C}=\mathrm{C}$ bond, rotation around it is very difficult (the height of the energy barrier $\Delta E$ is about $270 \mathrm{~kJ} / \mathrm{mol}$ ) and $A=334.5$.

Coefficient $B=\left(K_{F}-K_{E}\right) \tau_{0}^{2} J^{-1}$ depends on the moment of forces, created by the interaction of the $i$-th $\mathrm{CH}_{2}$-group and hydrocarbon chains of neighboring lipid molecules, rotating around the OX axis (see Fig. 4). Taking into account the estimates given above, $B=0.1154$.

The equations system (9) was solved by the fourth order Runge-Kutta method. The initial angle $\Psi_{10}$ and the corresponding angular velocity $\omega_{10}$ were set as the initial state of the system for the first link. The rest of links at the initial moment of time had zero angle of rotation and angular velocity. Since a finite chain was considered, boundary links (zero and $(n+1)$-th were introduced, which parameters values were fixed.

The evolution of a hydrocarbon chain, consisting of $20 \mathrm{CH}_{2}$-groups, is shown in Fig. 6. This evolution is described by the rotation angle $\Psi$ (radians) of each of the groups. Here, the group number is indicated along the abscissa axis, time was measured in times $\tau_{0}$, and the constants in eq. (9) were equal to $A=15.5$ and $B=0.1154$. A kink forms in the hydrocarbon chain at time $\tau=0.5$, which further transforms into a kink-soliton, spreading along the chain at $V$ velocity. Thus, the kink-soliton is formed in the hydrocarbon chain, moving at a given velocity along it. The deformation process of the erythrocyte membrane during its passage through the microcapillary layer and through the lungs alveoli is periodic. The kink-soliton is formed periodically in the considered hydrocarbon chain.

Coefficient $B$ in eq. (9) is determined by coefficients $K_{F}$ and $K_{E}$, which depend on the membrane conformation. With some change in the composition of membrane lipids, for example, with an increase or decrease in cholesterol concentration, the $B$ coefficient will change. It is important to understand how this can affect the formation and motion of the kink-soliton. In order to find out, solutions of eq. (9) were constructed for a hydrocarbon chain of 20 groups at a fixed coefficient $A=15.5$. Coefficient $B$ varied from 0.1 to 0.35 . It was found that the kink-soliton appeared in the indicated range, its energy did not depend on the coefficient $B$. This was consistent with formula (8), indeed, if we substitute the definition of $A$ coefficient in it, we get the following value $B=(2.772 J l A) /\left(\tau_{0}^{2} h\right)$.

It is important to understand what happens when the coefficient $A$ changes. For this purpose, parametric calculations were carried out with the constant coefficient $B=0.11544$, where $A$ varied from 15.5 to 45 . It was found that 


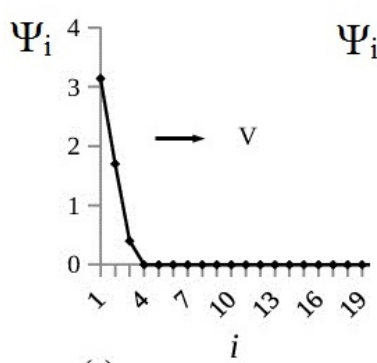

(a)

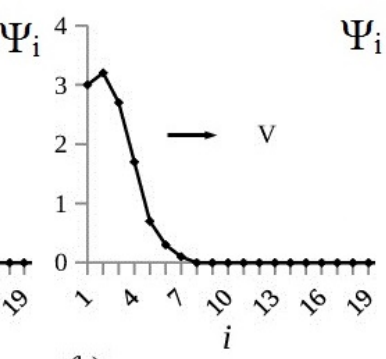

(b)

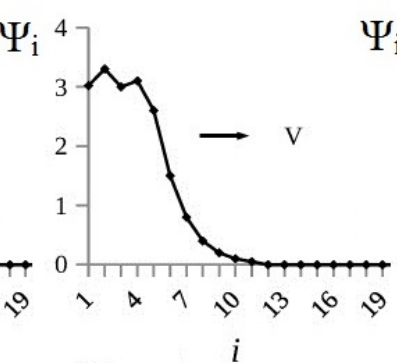

(c)

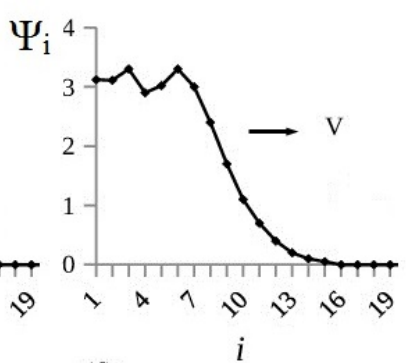

(d)

FIG. 6. Evolution of the rotation angle of the hydrocarbon chain $\mathrm{CH}_{2}$-groups at different times $\tau=0.5,1,1.5,2$ (from left to right)

a kink-soliton always appeared in this case as well. The dependence of the dimensionless value of the kink-soliton energy $W_{A}=W / W_{i}$, where $W_{i}$ is the kink-soliton energy at $A=15.5$, is shown in Fig. 7. The energy of a soliton is directly proportional to the coefficient $A$, which agrees with formula (8).

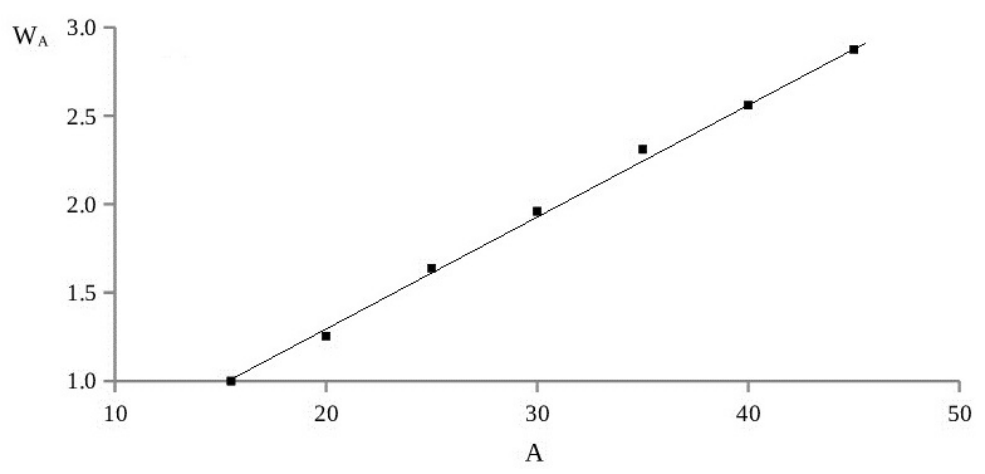

FIG. 7. Dependence of the kink-soliton dimensionless energy $W_{A}$ on the coefficient $A$

In phospholipid molecules, along with saturated $\mathrm{C}-\mathrm{C}$ bonds, there can be links with a $\mathrm{C}=\mathrm{C}$ double bond. The modeling has shown that, the kink-soliton is also formed in the presence of such bonds, but its structure and properties change significantly. A comparison of the kink-soliton's energy distribution over individual groups is shown in Fig. 8. Here the values of the relative energy $W / W_{m}$ ( $W$ is the rotational motion energy of the $i$-th $\mathrm{CH}_{2}$-group, $W_{m}$ is the maximum energy of the fourth $\mathrm{CH}_{2}$-group rotational motion) of the rotational motion of each $\mathrm{CH}_{2}$-group saturated (Fig. 8a) and unsaturated (Fig. 8b) a hydrocarbon chain during a soliton passing along it are given.
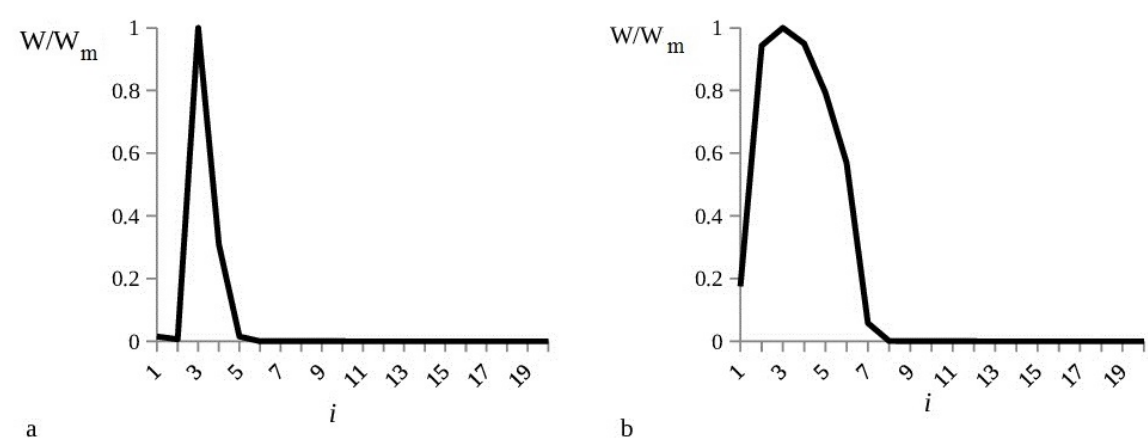

FIG. 8. Energy of each saturated $\mathrm{CH}_{2}$-group rotational motion (a) and unsaturated (b) hydrocarbon chain during a soliton passing along it

The links $2,3,4$ are with a double bond $\mathrm{C}=\mathrm{C}$ in the unsaturated chain, the rest is with a single $\mathrm{C}-\mathrm{C}$ bond. The presented picture corresponds to the time $\tau=1$ (see Fig. 6b), when the link number 4 has the highest angular velocity (and kinetic energy). The group number is again plotted along the abscissa axis. In the presence of a single bond, a 
soliton is actually formed by four groups 3, 4, 5, 6 (see Fig. 6b). Group 4 has the maximum energy, the energy of the 5th group is about $30 \%$ of the maximum, and groups 3 and 6 have very low relative energies. On the other hand, in the presence of double bonds, groups 1,2,6,7 also have a sufficiently high energy. The kink-soliton is formed not by four, but by five groups.

The difference between a soliton in an unsaturated hydrocarbon chain and a soliton in a saturated chain is that, the $\mathrm{CH}_{2}$-groups, connected by a double bond continue to oscillate with large amplitude, taking up part of the soliton energy after the soliton has passed the front. This is not observed in the saturated chain; after the soliton passes the front, the $\mathrm{CH}_{2}$ groups vibrate with very small amplitude. Thus, the soliton is "blurred" in the unsaturated chain, and it is necessary to give the former greater energy for its existence. A stronger transverse compression of the erythrocyte membrane is necessary during the erythrocyte passaging through the microcapillaries. In this case, in the erythrocyte membrane from saturated and unsaturated phospholipids, kink-solitons will appear in saturated chains, and will not do it in unsaturated chains. Therefore, gaseous transport through the membrane should decrease with an increase in the concentration of unsaturated lipids in it. It is known that when animals hibernate, the lipid bilayer of the erythrocyte membranes is enriched with lipids with unsaturated bonds in hydrocarbon chains. According to the developed model, this contributes to a decrease in oxygen permeability for these membranes.

\section{Discussion of the obtained results and concluding remarks}

Let us consider the above approximations, before discussing the obtained results. Eqs. (2) and (7) describe the interactions between hydrocarbon chain links of phospholipids in the lipid bilayer of the erythrocyte membrane approximately. First, the interaction of the lipid hydrocarbon chain links with membrane proteins is not considered. Meanwhile, membrane proteins make up approximately $50 \%$ of the erythrocyte membranes mass; they strongly affect the conformation of the membranes lipid bilayer [20]. Secondly, considering the forces moment $\mathbf{N}_{i 3}$ in eq. (1), we neglected the steric interaction of hydrogen atoms during rotation of the $i$-th $\mathrm{CH}_{2}$-group with groups $(i+2)$, and $(i-2)$, etc., leaving only the moment of forces, created due to the steric interaction of hydrogen atoms during the rotation of two neighboring $\mathrm{CH}_{2}$-groups $\left(i\right.$-th and $(i+1)$-th) and two neighboring $\mathrm{CH}_{2}$-groups $(i$-th and $(i-1)$-th) around $\mathrm{C}-\mathrm{C}$ bonds. Meanwhile, more distant $\mathrm{CH}_{2}$-groups also affect the rotation of the $i$-th $\mathrm{CH}_{2}$-group. This error is easy to estimate. The steric interaction of hydrogen atoms is due to Van Der Waals forces (dipole-dipole induced interaction) between them. These forces are inversely proportional to the distance between atoms to the sixth power. Therefore, the exact value of $\chi_{e}$ coefficient in eq. (2) is: $\chi_{e}=\chi\left(1+2^{-6}+3^{-6}+\ldots\right)$. The difference between the exact value of this coefficient and that used in eqs. (2) and (7) is less than $2 \%$. Thus, the model, developed here, is quite adequate. In principle, of course, it is not difficult to take into account subsequent contributions to $\chi_{e}$, however this will not lead to significant changes in the final results.

Now, let us consider the possibilities of the kink-soliton model for describing processes actually observed in practice. With an increase in cholesterol concentration in biomembranes, their permeability to oxygen molecules decreased. This was established by the method of molecular dynamics [21] and by measuring the biomembrane permeability of a human eye's corneal cells [22-26], the Chinese hamster's ovary cells [27] using electron paramagnetic resonance. These data are quite explainable by the kink-soliton model of oxygen molecule transport. Indeed, cholesterol molecules can easily move along the membrane, being incorporated into the biomembrane, increasing the interaction energy between the hydrocarbon chains of the bilayer molecules and creating mechanical compressive stresses in the membrane. The chains are in the trans-configuration, and their transition to the gauche-trans-gauche-configuration turns out to be difficult (Fig. 2). Cholesterol hindered the appearance of kinks-solitons, and the membrane permeability decreased, coming up to the hydrocarbon chains of phospholipids.

After all, it was experimentally established that the oxygen permeability was lower in the protein-lipid domains of cytoplasmic membranes than in the areas between them [27]. The same fact was found for artificial lipid membranes, enriched with cholesterol [28]. This difference can be explained by the fact that mechanical compression deformations prevail in the protein-lipid domains of biomembranes, and tensile deformations prevail in the areas between them [20]. The kinks-solitons formation is hindered in protein-lipid domains, while the kinks-solitons are more often formed in the regions between them and the oxygen permeability for these regions is higher.

Further, it was found that by using the electron paramagnetic resonance method, the inclusion of a cis- or transdouble bond in the C9-C10 position of the alkyl chain reduced oxygen transfer at all sites of the membrane in the absence of cholesterol [14]. If oxygen were transported through the membrane only due to passive diffusion, then the presence of a double bond in the alkyl chain should have increased the oxygen transfer. Double bonds created kinks in the alkyl chain; the lipid bilayer was more friable than the lipid bilayer of phospholipids with saturated bonds in the alkyl chain. Passive diffusion through a more friable lipid bilayer should be higher, but the experiment showed the opposite fact. The model of oxygen molecule transportation using kinks-solitons, presented in this paper, explains the results of this experiment. Turning around is facilitated (the energy barrier height is about $12.5 \mathrm{~kJ} / \mathrm{mol}$ ) for a link 
with a saturated $\mathrm{C}-\mathrm{C}$ bond. On the other hand, for a link with a $\mathrm{C}=\mathrm{C}$ double bond, such a turn is difficult, on the contrary (the height of the energy barrier is about $270 \mathrm{~kJ} / \mathrm{mol}$ of energy). Therefore, the frequency of the kink-soliton occurrence becomes very low. It is known that when fish start hibernation, the lipid bilayer of the plasma membranes of their cells is enriched with phospholipids with unsaturated bonds in hydrocarbon chains [29]. This helps to reduce the oxygen permeability of these membranes.

Mechanical stresses in membranes affect the ability of erythrocytes to fold when they pass through micro capillaries [30]. These stresses change under the influence of exogenous and endogenous factors: hormones [31, 32], nanoparticles [33-36], blood plasma proteins [37], vitamin E [38]. Apparently, the change in the microviscosity of the erythrocyte membranes is one of the mechanisms of a human body adaptation to the harsh conditions of the Far North. The native membrane of an erythrocyte in blood undergoes a structural transition under physiological parameters of blood [39]. Small changes in blood $\mathrm{pH}$, hormone concentration, and temperature dramatically change the biomembranes conformation, thus altering its functions by changing the field of mechanical stress in the biomembrane [39,40]. The model of the kinks-solitons in the lipid bilayer developed corresponds to this general principle. A slight increase in the transverse mechanical pressure on the membrane causes a change in its structure and a sharp increase in the diffusion of gas molecules through it. The existence of kinks-solitons in a biomembrane can apparently be detected by the nuclear magnetic resonance method [41].

Longitudinal mechanical compressive stresses prevail in the erythrocyte membranes, when they pass through arteries and veins, which diameter is larger than erythrocytes [19,31]. There are no conditions for the kinks-solitons formation. The membrane permeability is low for gas molecules.

On the contrary, when the erythrocyte passes through the microcapillary bed, for which the diameter is smaller than the diameter of red blood cells, it folds and the erythrocyte membrane is affected by both transverse compression and longitudinal mechanical tension (see Fig. 2). In addition, contractions of smooth muscle cells of microcapillaries occur with a high frequency, leading to radial mechanical displacements of the microvessels walls $[32,42,43]$. There are the conditions for the membrane to shrink to a critical value $\Delta$, the kinks-solitons begin to run along the hydrocarbon tails of phospholipids, moving gas molecules with their bends. The permeability of the erythrocyte membrane increases sharply.

According to the developed model, the biomembrane electric field has very little effect on the kinks-solitons appearance, since $K_{E} \ll K_{F}$. But, what else can affect the passage of the erythrocytes through capillaries on the gas molecules transport through the membrane? The microcapillary diameter is often less than the diameter of an erythrocyte. The erythrocyte folds into a tube and passes through the capillary. In this case, the boundary layer of immiscible water adjacent to the membrane from the outside is almost completely stripped off the erythrocyte surface. The total permeability for gas molecules from the erythrocyte and into the erythrocyte increases. At the same time, a slight decrease in blood plasma $\mathrm{pH}$ occurs in the peri-membrane layer. Red blood cells receive additional force pushing them along the microcapillary layer [44].

\section{Acknowledgements}

This work was financially supported by the Russian Foundation for Basic Research (Grant No. 19-01-00399).

\section{References}

[1] Bahri M.A., Heine B.J, Hans P., et al. Quantification of lipid bilayer effective microviscosity and fluidity effect induced by propofol. Biophys. Chem., 2005, 114, P. 53-61.

[2] Kreuzer F., Yahr W.Z. Influence of red cell membrane on diffusion of oxygen. J. Appl. Physiol., 1960, 15, P. 1117-1122.

[3] Blank M. Monolayer permeability and the properties of natural membranes. J. Phys. Chem., 1962, 66 (10), P. 1911-1918.

[4] Ivanov I.I., Loktyushkin A.V., et al. Oxygen channels of the erythrocyte membrane. Doklady biochemistry and biophysics, 2007,414 (1), P. $137-140$.

[5] Denker B.M., Smith B.L., Kuhajda F.P., Agre P. Identification, purification, and partial characterization of a novel Mr 28,000 integral membrane protein from erythrocytes and renal tubules. J. Biol. Chem., 1988, 263 (30), P. 15634-15642.

[6] Preston G.M., Carroll T.P., Guggino W.B., Agre P. Appearance of water channels in Xenopus oocytes expressing red cell CHIP 28 protein. Science, 1992, 256 (5055), P. 385-387.

[7] Saparov S.M., Liu K., Agre P., Pohl P. Fast and selective ammonia transport by aquaporin-8. J. Biol. Chem., 2007, 282 (8), P. $5296-5301$.

[8] Endeward V., Cartron J.P., Ripoche P., Gros G. RhAG protein of the Rhesus complex is a $\mathrm{CO}_{2}$ channel in the human red cell membrane. FASEB J., 2008, 22 (1), P. 64-73.

[9] Agre P., Preston G.M., et al. Aquaporin CHIP: the archetypal molecular water channel. Am. J. Physiol., 1993, 265 (4), P. $463-476$.

[10] Cooper G.J., Boron W.F. Effect of PCMBS on $\mathrm{CO}_{2}$ permeability of Xenopus oocytes expressing aquaporin 1 or its C189S mutant. Am. $J$. Physiol., 1998, 275 (6), P. 1481-1486.

[11] Endeward V., Musa-Aziz R., et al. Evidence that aquaporin 1 is a major pathway for $\mathrm{CO} 2$ transport across the human erythrocyte membrane. FASEB J., 2006, 20 (12), P. 1974-1981.

[12] Endeward V., Gros G. Low carbon dioxide permeability of the apical epithelial membrane of guinea-pig colon. J. Physiol., $2005, \mathbf{5 6 7}$ (1), P. $253-265$. 
[13] Träuble H. The movement of molecules across lipid membranes: A molecular theory. J. Membr. Biol., 1971, 4 (1), P. 193-208.

[14] Subczynski W.K., Hyde J.S., Kusumi A. Effect of alkyl chain unsaturation and cholesterol intercalation on oxygen transport in membranes: a pulse ESR spin labeling study. Biochemistry, 1991, 30 (35), P. 8578-8590.

[15] Flory P.J., Volkenstein M. Statistical mechanics of chain molecules. Biopolymers, 1969, 5, P. 699-700.

[16] Secomb T.W., Pries A.R. Blood viscosity in microvessels: experiment and theory.Comptes Rendus Physique, 2013,14 (6), P. $470-478$.

[17] Rubin A.B. Biophysics, Nauka, Moscow, 2004, 469 p.

[18] Rudyak V.Ya. Statistical Aerohydromechanics of Homogeneous and Heterogeneous Media. Vol. 2. Hydromechanics, NSUACE, Novosibirsk, $2005,468 \mathrm{p}$.

[19] Dodd R.K., Eilbeck J.C., Gibbon J.D., Morris H.C. Solitons and Nonlinear Wave Equations. Academic Press, New York, 1982,697 p.

[20] Panin L.E., Mokrushnikov P.V., Kunitsyn V.G., Zaitsev B.N. The interaction mechanism of cortisol and catecholamines with structural components of erythrocyte membranes. Journal of physical chemistry B, 2010, 114, P. 9462-9473.

[21] Angles G., Dotson R., Bueche K., Pias S.C. Predicted Decrease in Membrane Oxygen Permeability with Addition of Cholesterol. Adv. Exp. Med. Biol., 2017, 977, P. 9-14.

[22] Subczynski W.K., Widomska J., Mainali L. Factors Determining the Oxygen Permeability of Biological Membranes: Oxygen Transport Across Eye Lens Fiber-Cell Plasma Membranes. Adv. Exp. Med. Biol., 2017, 977, P. 27-34.

[23] Widomska J., Raguz M., Subczynski W.K. Oxygen permeability of the lipid bilayer membrane made of calf lens lipids. Biochim. Biophys. Acta, 2007, 1768 (10), P. 2635-2645.

[24] Subczynski W.K., Widomska J., Feix J.B. Physical properties of lipid bilayers from EPR spin labeling and their influence on chemical reactions in a membrane environment. Free Radic. Biol. Med., 2009, 46 (6), P. 707-718.

[25] Subczynski W.K., Raguz M., et al. Functions of cholesterol and the cholesterol bilayer domain specific to the fiber-cell plasma membrane of the eye lens. J. Membr. Biol., 2012, 245 (1), P. 51-68.

[26] Mainali L., Raguz M., O’Brien W.J., Subczynski W.K. Properties of membranes derived from the total lipids extracted from the human lens cortex and nucleus. Biochim. Biophys. Acta, 2013, 1828 (6), P. 1432-1440.

[27] Khan N., Shen J., et al. Plasma membrane cholesterol: a possible barrier to intracellular oxygen in normal and mutant CHO cells defective in cholesterol metabolism. Biochemistry, 2003, 42 (1), P. 23-29.

[28] Kawasaki K., Yin J.J., et al. Pulse EPR detection of lipid exchange between protein-rich raft and bulk domains in the membrane: Methodology development and its application to studies of influenza viral membrane. Biophys. J., 2001, 80 (2), P. 738-748.

[29] Mokrushnikov P.V., Panin L.E., et al. Interaction of corundum and quartz nanocrystals with erythrocyte membranes. Biophysics, 2011, 56 (6), P. 1074-1077.

[30] Kozelskaya A.I., Panin A.V., et al. Morphological changes of the red blood cells treated with metal oxide nanoparticles. Toxicology in Vitro, 2016, 37, P. 34-40.

[31] Panin L.E., Mokrushnikov P.V., Kunitsyn V.G., Zaitsev B.N. Interaction mechanism of anabolic steroid hormones with structural components of erythrocyte membranes. Journal of physical chemistry B, 2011, 115, P. 14969-14979.

[32] Bagayev S.N., Zakharov V.N., et al. Transcapillary exchange regulation by pulse blood pressure. Russian Journal of Biomechanics, 2008, 12 (3), P. 7-14.

[33] Mokrushnikov P.V., Dudarev A.N., et al. Effects of native and oxidized apolipoprotein A-I on lipid bilayer microviscosity of erythrocyte plasma membrane. Journal Biochemistry (Moscow), Supplement Series A: Membrane and Cell Biology, 2017,11 (1), P. 48-53.

[34] Mokrushnikov P.V. A Mechanism of the Interaction of Metal Oxide Nanoparticles with Biological Membranes. Biophysics, 2020, 65 (1), P. 65-69.

[35] Popov A.L., Savintseva I.V., et al. Cytotoxicity analysis of gadolinium doped cerium oxide nanoparticles on human mesenchymal stem cells. Nanosystems: physics, chemistry, mathematics, 2018, 9 (3), P. 430-438.

[36] Popov A.L., Savintseva I.V., et al. PVP-stabilized tungsten oxide nanoparticles $\left(\mathrm{WO}_{3}\right)$ nanoparticles cause hemolysis of human erythrocytes in a dose-dependent manner. Nanosystems: physics, chemistry, mathematics, 2019, 10 (2), P. 199-205.

[37] Poteryaeva O.N., Russkikh G.S., et al. Hormonal status, lipid profile and membranes microviscosity of north residents. Journal of Ural Medical Academic Science, 2014, 2 (48), P. 149-152.

[38] Mokrushnikov P.V., Osipova L.P., Gol'tsova T.V., Rozumenko A.A. Erythrocyte membranes microviscosity in the population of Samburg village Yamalo-Nenets Autonomous Okrug. Yakut medical journal, 2016, 2 (54), P. 15-16.

[39] Mokrushnikov P.V., Panin L.E., et al. Structural transitions in erythrocyte membranes (experimental and theoretical models). NGASU, Novosibirsk, 2019, 286 p.

[40] Mokrushnikov P.V. Mechanical Stresses in the Lipid Bilayer of Erythrocyte Membranes. In: "Lipid Bilayers: Properties, Behavior and Interactions" edited by Mohammad Ashrafuzzaman, Nova Science Publishers, NY, 2019, P. 43-91.

[41] Filippov A., Oradd G., Lindblom G. Lipid lateral diffusion in ordered anddisordered phases in raft mixtures. Biophys. J., 2004, 86, P. 891-896.

[42] Bagayev S.N. Fomin Yu.N., et al. Investigation of transcapillary exchange by the laser method. Laser Physics, 2005,15 (9), P. $1292-1298$.

[43] Bagaev S.N., Zakharov V.N., et al. Investigation of the physical mechanisms of blood microcirculation and transcapillary exchange using a phase-sensitive laser method. Russian Journal of Biomechanics, 2006, 10 (3), P. 22-40.

[44] Mokrushnikov P.V. The influence of $\partial \mathrm{H}$ on the surface tension of suspended erythrocytes. The Siberian Scientific Medical Journal, 2010, 1, P. $38-46$. 